\title{
Array Antenna Feeding Network Design for 5G MIMO Applications
}

\author{
Nurhan Türker Tokan (iD) \\ Department of Electronics and Communications Engineering, YıldizTechnical University, İstanbul, Turkey
}

Cite this article as: Türker Tokan N. Array Antenna Feeding Network Design for 5 G MIMO Applications. Electrica, 2019; 19(2): 120-127.

\begin{abstract}
Emerging smart antenna systems require different beam patterns of multiple antennas. Although adjustable phase shifters are mostly used in continuousbeam systems, the Butler matrix is used in switching-beam systems due to its low cost and easy fabrication. In this study, an antenna-array-feeding circuit based on the Butler matrix that can be used for Multiple Input Multiple Output applications is designed for 5G new radio. With the proposed switching system, the control of four beams can be achieved. The Butler circuit, designed to cover the 3.5-4.2 GHz $5 \mathrm{G}$ band, has a low complexity and is capable of meeting the need for high data throughput. A simulation of the circuit and circuit sub-elements designed using a 0.508-mm-thick substrate material is performed using the Computer Simulation Technology Microwave Studio computer-aided design tool. Furthermore, a prototype of the Butler circuit is fabricated, and the amplitude and phase variations at the output ports are measured. An average transmission loss of the feed circuit is measured as $1.5 \mathrm{~dB}$, and when the length of the Phase Shifter in the circuit is set to $\lambda / 8$, with a four-element linear array added to the output of the Butler circuit, the main beam is steered to \pm 150 and \pm 350 having maximum gain in the 6.39-8.77 dBi range.
\end{abstract}

Keywords: 5G, antenna array, Butler circuit, MIMO, Vivaldi

\section{Corresponding Author: \\ Nurhan Türker Tokan}

E-mail:

nturker@yildiz.edu.tr

Received: 08.02 .2019

Accepted: 11.06 .2019

C Copyright 2019 by Electrica Available online at

http://electrica.istanbul.edu.tr

DOI: 10.26650/electrica.2019.19004

Content of this journal is licensed under a Creative Commons Attribution-NonCommercial 4.0 International License.

\section{Introduction}

Wide bandwidths are critical in modern wireless communication applications. Today, communication systems such as LTE need broadband operation for a high data rate transmission. Broadband LTE consists of multiple narrow-band signals. Multiple input-multiple output (MIMO) systems have recently been used in modern wireless systems. These systems use the spatial diversity technique consisting of multiple transmitter and receiver antennas. With this technique, the channel fading effects are minimized, and the channel capacity is increased [1-2].

The transition from $4 \mathrm{G}$ to $5 \mathrm{G}$ mobile network is expected to occur in the next 10 years $[3,4]$. Due to constantly increasing network speed and capacity requirements, research has been conducted in many countries to implement the $5 \mathrm{G}$ wireless broadband technology. The wide range of services, frequencies, and application areas make $5 \mathrm{G}$ new radio precious. In addition to low and high frequencies, medium frequencies $(1 \mathrm{GHz}-6 \mathrm{GHz})$ are planned to be used as well. Within this band, the $3.5-4.2 \mathrm{GHz}$ range is defined as the new radio $5 \mathrm{G}$ medium band, and it includes both licensed and unlicensed frequency bands.

Multi-beam antennas help to improve the transmission quality and increase the channel capacity by reducing the interference and multi-path fading in wireless communication systems. The beam-shaping techniques that provide unique beams for beam switching or beam steering have become an indispensable element of intelligent antenna systems. The Blass [5, 6], Nolen $[7,8]$, and Butler matrices $[9,10]$ are analog solutions that generate multiple beams by selecting input excitation. Among them, the Butler matrix is a widely used beam former for the antenna array applications, such as multi-beam antenna systems. Butler circuits have been used in many beam-switching array systems due to their simple design, low cost, and easy fabrication [11-14]. The size of branch-line couplers in the Butler circuit makes its incorporation with monolithic mi- 
crowave integrated circuits complicated and expensive. To overcome the size disadvantage of branch-line coupler, a number of approaches have been proposed [15-17].

In this paper, an antenna-array-feeding network based on the Butler matrix is designed for MIMO applications of $5 \mathrm{G}$ new radio. The Butler matrix is used to shape the beam in a phased array antenna system. $N$ beams are generated by feeding $N$ antennas with a Butler network having an $N$ input- $N$ output. Since $90^{\circ}$ hybrid couplers are used in the Butler network, complexity increases with an increase in the number of beams. Therefore, the most commonly used structure to obtain an acceptable beam scanning angle is $4 \times 4$ Butler network. It is possible to direct the main beam to $\pm 45^{\circ}$ and $\pm 15^{\circ}$ by placing radiating elements with a half-wavelength distance. It is also possible to direct the beam to a different angular direction by controlling input phases and distance between the elements. The spatial resolution can be improved by increasing the number of beams. For this, a higher order Butler matrix should be used $(\mathrm{N}=8,16, \ldots)$. However, an increase in the degree of the Butler matrix will lead to a higher power loss. Moreover, the requirement of extra crossover elements in the network will make the design difficult.

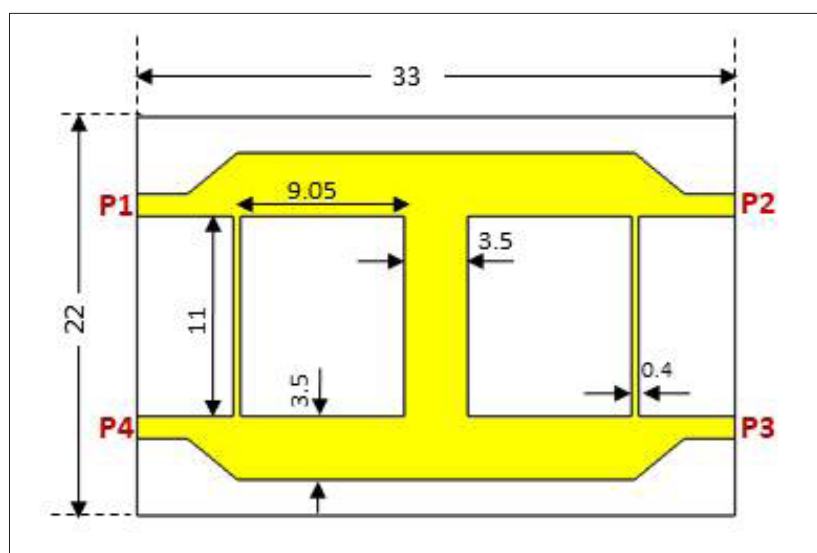

Figure 1. Wideband $3 \mathrm{~dB}$ hybrid coupler

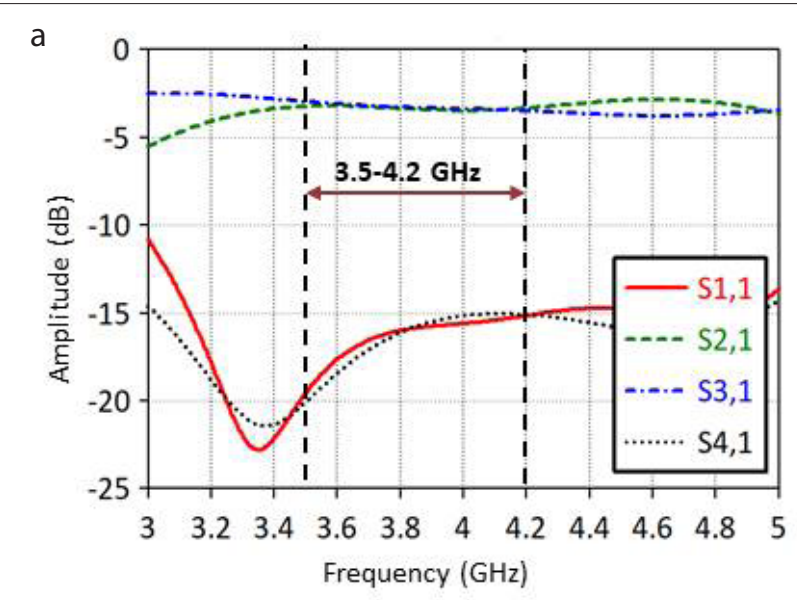

The Butler circuit, designed to operate in the 3.5-4.2 GHz 5G band, is capable of meeting the need for high data throughput [18]. In this study, a wideband $4 \times 4$ Butler circuit is designed for medium frequencies of $5 \mathrm{G}$ new radio. In Section 2, the designed Butler circuit and structures used in the circuit are introduced. Simulation results of wideband hybrid coupler and crossover elements are also given in this section. All simulations are performed using the CST Microwave Studio (CST $\mathrm{GmbH}$, Darmstadt, Germany.), a computer-aided design tool using a finite-integration technique. The measurement results of the designed circuit are given in Section 3. In Section 4, the beam-steering capability of the Butler circuit is demonstrated with an application example based on a four-element linear antenna array.

\section{Butler Matrix Design}

The $4 \times 4$ Butler matrix consists of two wideband crossover, four wideband $3 \mathrm{~dB}$ hybrid couplers, and four phase shifters. Rogers 3003 with a thickness of $0.508 \mathrm{~mm}$ and a dielectric constant of $\varepsilon_{r}=3$ is used as substrate material. The conductive material on both sides of the dielectric is copper with a thickness of $17 \mu \mathrm{m}$. In the design of the Butler circuit, microstrip transmission line is used. Input and output ports of the circuit are designed to be compatible with 50 .

Performance of the Butler matrix is directly related with the performance of the elements it contains. Thus, the simulation and optimization of each circuit element was made separately and then combined. The CST Microwave Studio was used for the optimization of the designed structures.

\section{Wideband Hybrid Coupler Design}

The coupler is the most important circuit element in the Butler circuit since there are four in the circuit and by cascading two of them, a crossover is formed. The bandwidth of a $90^{\circ}$ hybrid coupler can be increased by using two-section branch-line technique instead of the standard coupler architecture [19].

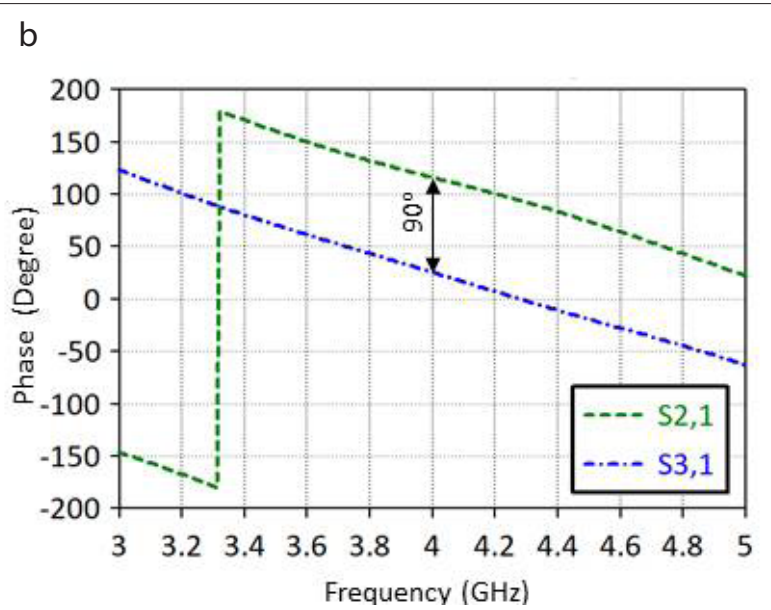

Figure 2. a, b. S-parameters of the hybrid coupler: (a) amplitude response and (b) phase response 
a

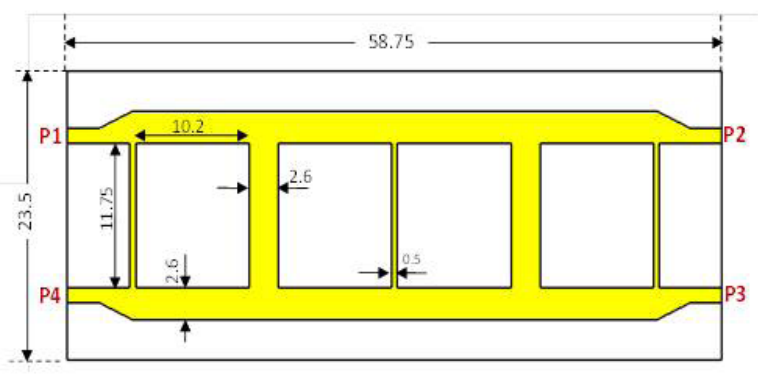

b

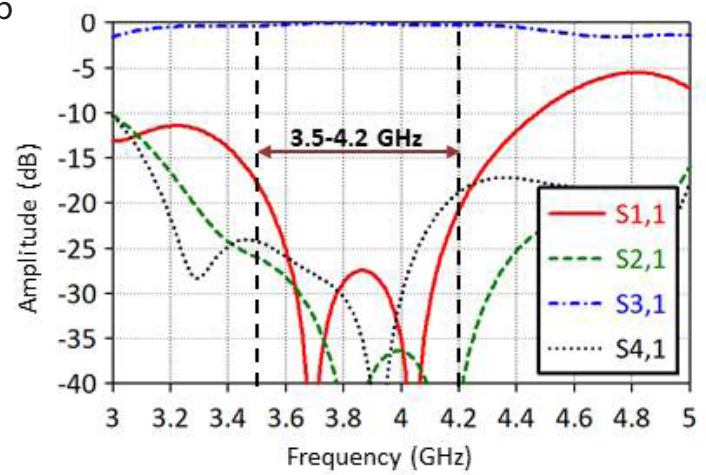

Figure 3. a, b. Wideband crossover: (a) top view and (b) amplitude response

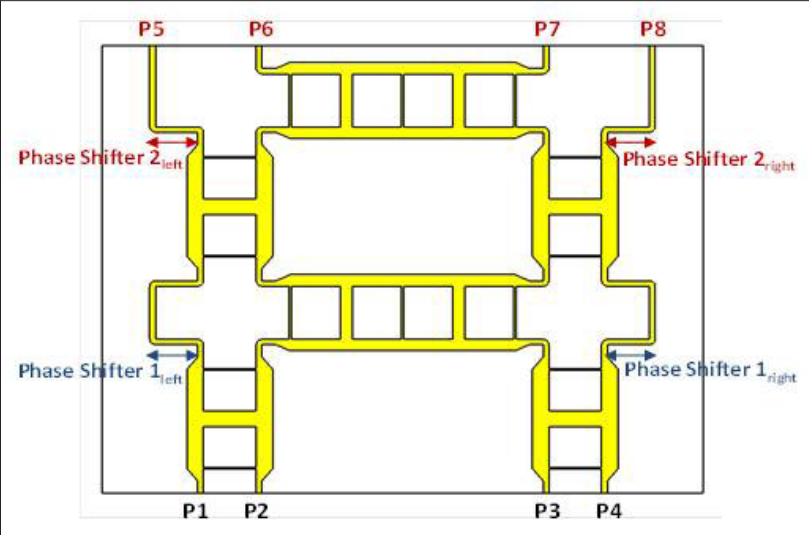

Figure 4. Wideband Butler matrix

Figure 1 is top view of the wideband $3 \mathrm{~dB}$ hybrid coupler designed for $5 \mathrm{G}$ medium frequencies. All dimensions are indicated on the figure. All units are in millimeters.

The strip width is set to $1.24 \mathrm{~mm}$ at the input-output ports to be compatible with $50 \Omega$. Ports of the symmetrical four-port structure are shown in Figure 1. While the narrow-band hybrid coupler contains $\lambda / 4$ transmission lines, line lengths obtained as a result of optimization for broadband operation are given in the figure. The width of the side lines are determined to have $Z_{o} / \sqrt{2}=35 \Omega$ characteristic impedance. For the transition, lines with $2 Z_{0} 2^{1 / 4}=118 \Omega$ characteristic impedance are used at sides, and $35 \Omega$ is used in the middle. The amplitude and phase response of S-parameters obtained when the circuit is fed through Port 1 is given in Figure 2. Due to the symmetrical structure of the circuit, results obtained from other ports are not shared.

In the 3.5-4.2 GHz band, power is divided evenly between the second and third port. The coupling parameter is approximately $3 \mathrm{~dB}$ throughout the entire band. The isolation at the forth port and return loss at the first port are below $15 \mathrm{~dB}$. Figure $2 \mathrm{~b}$ shows that the phase difference between the output ports (Ports 2 and 3 ) is $90^{\circ}$.

\section{Wideband Crossover}

A wideband crossover can be achieved by combining two wideband couplers [20]. A crossover is used at the intersection points to prevent combination of the signals. Thus, the main function of the crossover is to allow the signal flow to the transversal port and ensure isolation with adjacent ports. A wideband crossover structure was formed by combining the broadband $3 \mathrm{~dB}$ coupler introduced in Section 2.1. The crossover circuit is shown in Figure 3.a. The variation of S-parameters is given in Figure 3.b. It can be seen that the isolation between Port 1 and Port 2 is below 25 $\mathrm{dB}$, and the isolation between Port 1 and Port 4 is below $18 \mathrm{~dB}$. Furthermore, the coupling ratio between transverse ports (Port 1 and Port 3 ) is close to $0 \mathrm{~dB}$ throughout the entire band.

\section{Wideband Butler Matrix Design}

The $4 \times 4$ Butler circuit, which is formed by the use of four wideband hybrid power dividers, four phase shifters, and two wideband crossovers, is given in Figure 4. Phases of the output ports can be controlled by the use of two phase shifters (Phase Shifter $1_{\text {left' }}$ Phase Shifter $1_{\text {right }}$ ). In addition, positions and phases of the antenna array elements can be adjusted by the length of 50 transmission lines, leading to output ports (Phase Shifter $2_{\text {left' }}$ Phase Shifter $2_{\text {right }}$ ).

Figure 5.a shows the amplitude variation of the S-parameters for the wideband Butler circuit. The variation of output phases for the case when Phase Shifter $1_{\text {left }}=$ Phase Shifter $1_{\text {right }}=\lambda / 4$ is demonstrated in Figure 5.b. When the circuit is fed from Port 1, the isolation between Port 1 and other input ports (Ports 2-4) is below 14 $\mathrm{dB}$, and the coupling between Port 1 and output ports (Ports 4-8) is approximately $6 \mathrm{~dB}( \pm 1.5 \mathrm{~dB})$. Thus, the Butler circuit divides the transmitted input power to approximately four. Output phases vary linearly with frequency. Output phases can be controlled by using the phase shifters generated by transmission lines.

\section{Measurement Results}

To measure the amplitude and phase response of the Butler circuit, a prototype is fabricated with the LPKF ProtoMat $\mathrm{H} 100$ 
a

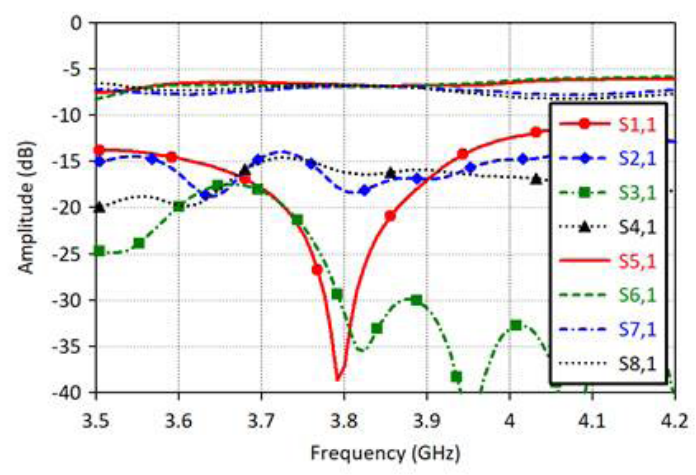

b

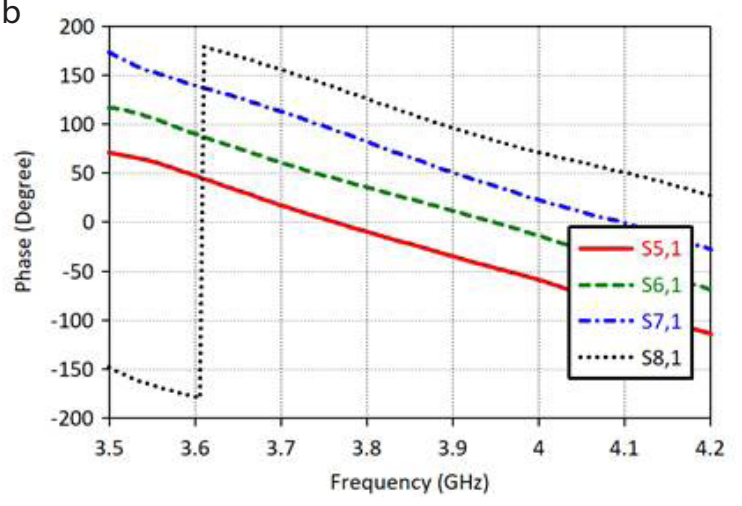

Figure 5. a, b. S-parameters of the Butler matrix fed from Port 1: (a) reflection coefficient from the input ports and coupling at the output ports, and (b) output phase variation example

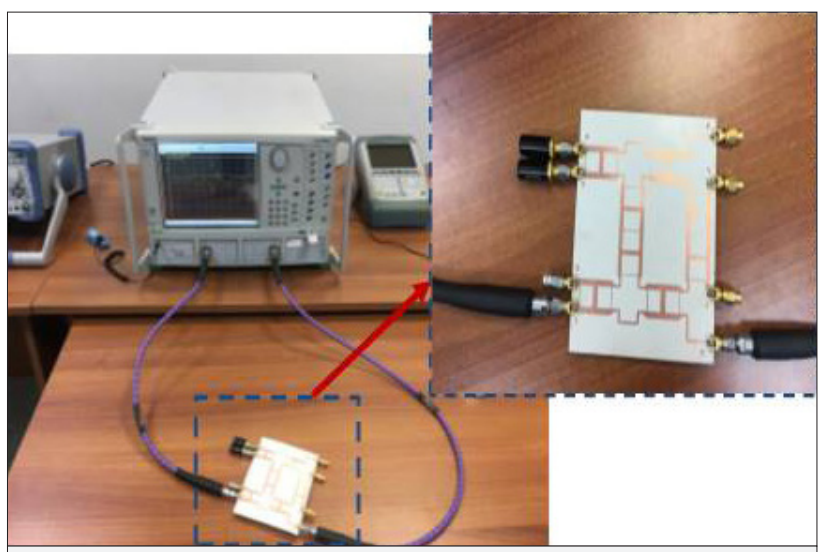

Figure 6. Wideband Butler matrix circuit measurement setup

printed circuit board production machine. The measurement setup is shown in Figure 6. An expanded view of the fabricated prototype is given in the inset. A SubMiniature version A (SMA) connector compatible with impedance is soldered to each port. S-parameters of the antenna are measured with the Anritsu vector network analyzer. During measurement, coaxial cables from the analyzer are connected to the two ports to be measured, while all other ports are terminated with matched loads to avoid reflection. Figure 7 shows return loss at the input ports and coupling at the output ports. Since the simulation results were given in the previous section, they are not shared with measurement results to prevent complexity. Due to the symmetrical structure of the circuit, similar results are obtained by feeding the circuit from Port 1 and Port 4 . Also, it is the case for Port 2 and Port 3. Thus, only the results obtained when the circuit is fed from Port 1 and Port 2 are included in Figure 7. The similarity between simulation and measurement results can be observed by comparing Figure 5 and 7.

\section{An Application Example: Vivaldi Array Fed by Butler Circuit}

In this section, an application example for the use of the Butler circuit for beam steering will be demonstrated. A four-element linear antenna array will be placed to the output ports of the
Butler circuit, and the main beam of the array will be steered to different angular directions with the excitation of input ports. For this purpose, a modified version of the commonly used Vivaldi antenna is used. A Vivaldi antenna is preferred due to its complete planar geometry and the possibility to feed it with a microstrip line.

\section{Modified Vivaldi Antenna Design}

The Vivaldi antenna is a widely used ultra-wideband antenna that has many practically applications. Due to its planar structure, it can be easily integrated with ultra-wideband sensor circuits. It has an almost symmetrical radiation pattern in E- and $\mathrm{H}$-planes. Theoretically, due to its exponentially tapered slotted structure, the Vivaldi antenna has unlimited bandwidth. However, in practice, it has a limited band as a result of its physical constraints, such as the expansion rate of the slot, the slot line width, and the transition from the feed line.

A well-known disadvantage of a classic Vivaldi antenna is its reduced directivity at low frequencies. By increasing the aperture size of the antenna, a better impedance matching and effective radiation can be achieved at low frequencies. Another solution is adding slots with varying lengths to increase the aperture of the antenna [21, 22]. The corrugated profile formed by adding slots to the two sides of the exponentially tapering provides a better characteristic (high gain, wider band) compared to the Vivaldi antenna, especially in microwave imaging applications [23]. For these reasons, a modified Vivaldi antenna with rectangular slots of varying lengths was used instead of a standard Vivaldi antenna.

The proposed Vivaldi antenna consists of a microstrip feed line, microstrip line to slot line transition, and the radiating structure. It is designed to operate as a receiver and transmitter in the $3.5 \mathrm{GHz}-12 \mathrm{GHz}$ (1: 3.4 bandwidth) frequency band. The slot curve of the Vivaldi antenna is an exponential function expressed by

$S(z)=\left(W_{\text {slot }} / 2\right) e^{a z}$, 
a

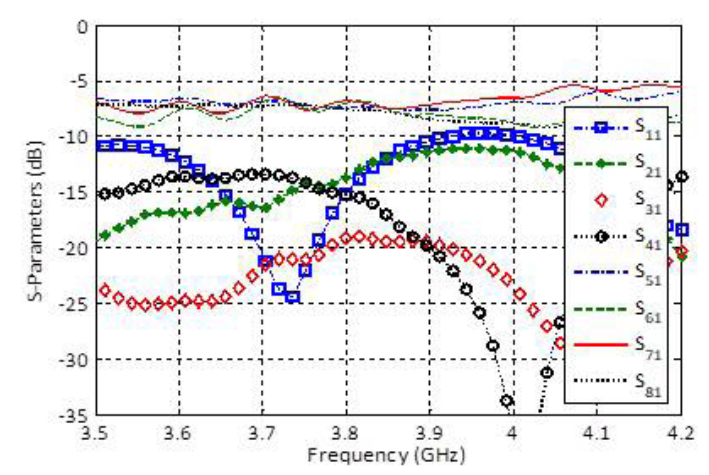

b

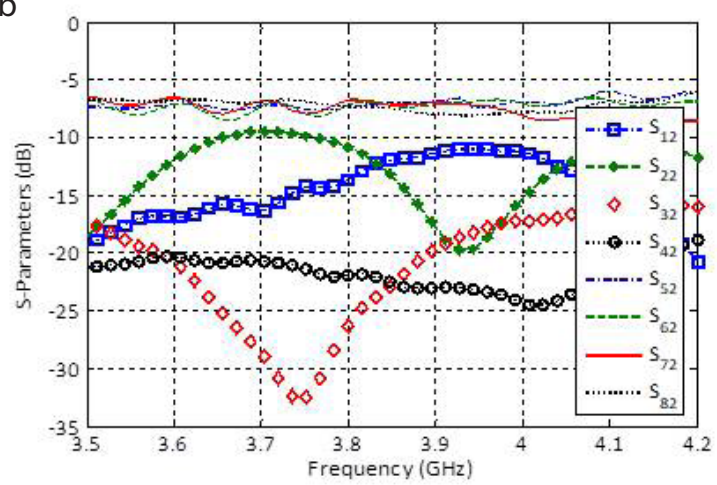

Figure 7. a, b. Measured S-parameters of the Butler matrix: (a) Port 1 as the input port and (b) Port 2 as the input port

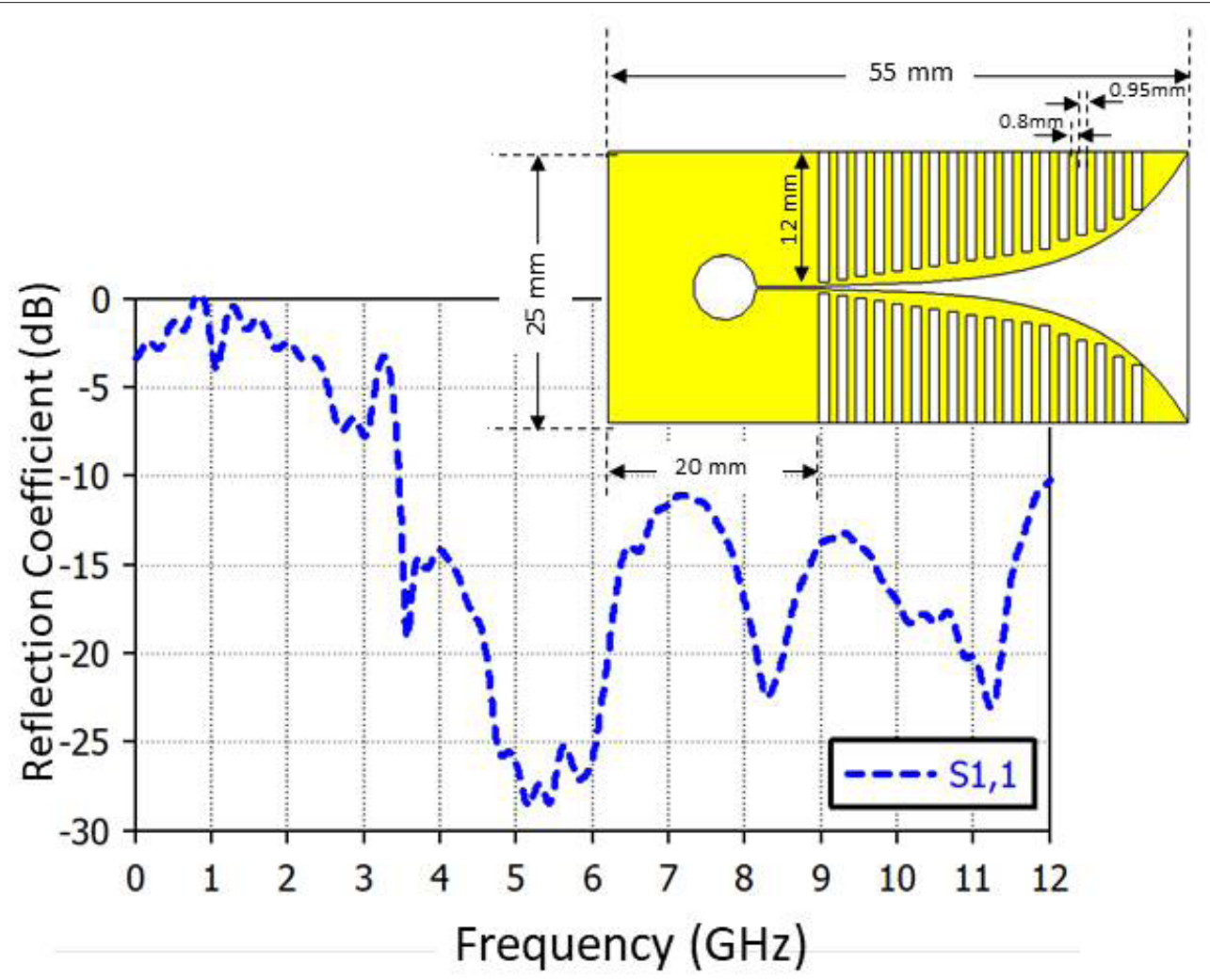

Figure 8. Reflection coefficient variation of the corrugated Vivaldi antenna

where $a=0.165$ and $W_{\text {slot }}=0.25 \mathrm{~mm}$. A quarter wavelength open circuit stub is used for wideband matching.

The size of the Vivaldi antenna is $25 \mathrm{~mm} \times 55 \mathrm{~mm}$. The design uses the Rogers 3003 laminate $\left(\varepsilon_{r}=3\right)$ with $0.508 \mathrm{~mm}$ substrate and $17 \mu \mathrm{m}$ copper. The distance between the rectangular slots, the width of the slots, and the length of the slots are also shown on the antenna. The distance between the slots and the width of the slots remain the same, whereas the length of the slots decreases toward the aperture. In simulations, it was observed that an increase in the number of slots changes the direction of the current at the edges and cause extra resonance. Slots at the edges act as a resistive load and contribute to the radiation by directing the wave to the slot line region. This improves the radiation characteristics of the Vivaldi.

The reflection behavior of the corrugated Vivaldi antenna is expressed in terms of S11. Figure 8 shows the variation of the reflection coefficient with frequency. Return loss is less than $10 \mathrm{~dB}$ in the frequency range $3.5 \mathrm{GHz}-12 \mathrm{GHz}$. The frequency band of the antenna includes the $3.5-4.2 \mathrm{GHz}$ band for the $5 \mathrm{G}$ 
medium frequencies. The structure of the modified Vivaldi antenna is shown in Figure 8 together with its dimensions as the inset. The change in the gain of the antenna within this band is $0.2 \mathrm{~dB}$. The gain pattern in $\mathrm{E}$ - and $\mathrm{H}$-planes at the center frequency (max. gain, $6.1 \mathrm{dBi}$ ) is shown in Figure 9.

\section{Four-Element Vivaldi Array Fed by the Butler Matrix}

Since the Butler circuit is an ideal feeding network for beamswitched array systems [11-14], it is designed for 5G medium frequencies as a feeding network for the four-element linear corrugated Vivaldi array. The distance between the elements is 25,60 , and $25 \mathrm{~mm}$, respectively. The transmission line at the output ports of the Butler circuit is in the microstrip form resulting in an uncomplicated connection with the Vivaldi antenna feed. Both the Vivaldi antenna and the Butler circuit use Rogers 3003 with $0.508 \mathrm{~mm}$ thickness. The ports are compatible with $50 \Omega$. In Fig. 10, the four-element Vivaldi array with the Butler feeding network is shown. The outer dimensions of the circuit are $135 \mathrm{~mm} \times 155 \mathrm{~mm}$.

Figure 11 shows the normalized radiation patterns of the corrugated Vivaldi array at $3.75 \mathrm{GHz}$ obtained by excitation of different input ports. Since the beam steering is at $\phi=90^{\circ}$ plane, only E-plane patterns are demonstrated. Asymmetry in the patterns is due to the balun used for impedance matching in the corru-

Table 1. Performance parameters of the corrugated Vivaldi array fed by the proposed Butler circuit

\begin{tabular}{lcccc}
\hline $\begin{array}{l}\text { Excitation } \\
\text { Port }\end{array}$ & $\begin{array}{c}\text { Main Beam } \\
\text { Direction } \\
\text { (o) }\end{array}$ & $\begin{array}{c}\text { Gain } \\
\text { (dBi) }\end{array}$ & $\begin{array}{c}\text { Directivity } \\
\text { (dB) }\end{array}$ & $\begin{array}{c}\text { dB } \\
\text { Beam- } \\
\text { width (o) }\end{array}$ \\
\hline P1 & 15 & 8.5 & 9.13 & 23.2 \\
\hline P2 & 35 & 6.86 & 7.47 & 27 \\
\hline P3 & 35 & 6.39 & 7.02 & 26 \\
\hline P4 & 15 & 8.77 & 9.39 & 23.1 \\
\hline
\end{tabular}

a

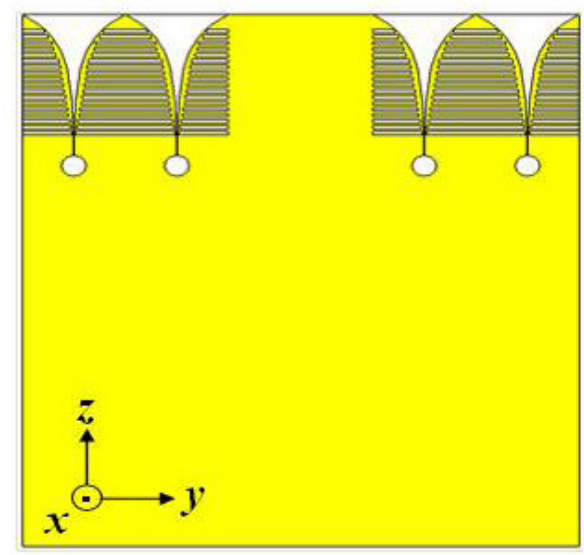

gated Vivaldi. Table 1 lists performance parameters of the radiation patterns. When the radiation patterns are observed, it can be seen that the main beam is steered to $\theta \cong+15^{0}$ and $-15^{0}$ and by the excitation of Ports 1 and 4, respectively and main beam is steered to and $\theta \cong+35^{0}$ and $-35^{0}$ by excitation of Ports 2 and 3 , respectively. Considering the $3 \mathrm{~dB}$ beam widths of the patterns given in Table 1, it can be concluded that with a four-element corrugated Vivaldi array fed by the proposed Butler circuit, half of the semi-sphere $\left(-45^{\circ} \leq \theta \leq 45^{\circ}\right)$ is scanned with a maximum gain of the pattern varying between 6.39 and $8.77 \mathrm{dBi}$.

\section{Conclusion}

MIMO systems with more than one user require a system that can create multiple beams in different directions. The Butler circuit is one of the most commonly used circuits for beam steering. In this work, a wideband Butler circuit compatible with planar geometry is introduced for beam-switching circuits to control the main beam direction. A $4 \times 4$ Butler circuit is designed for MIMO applications operating in $5 \mathrm{G}$ new radio me-

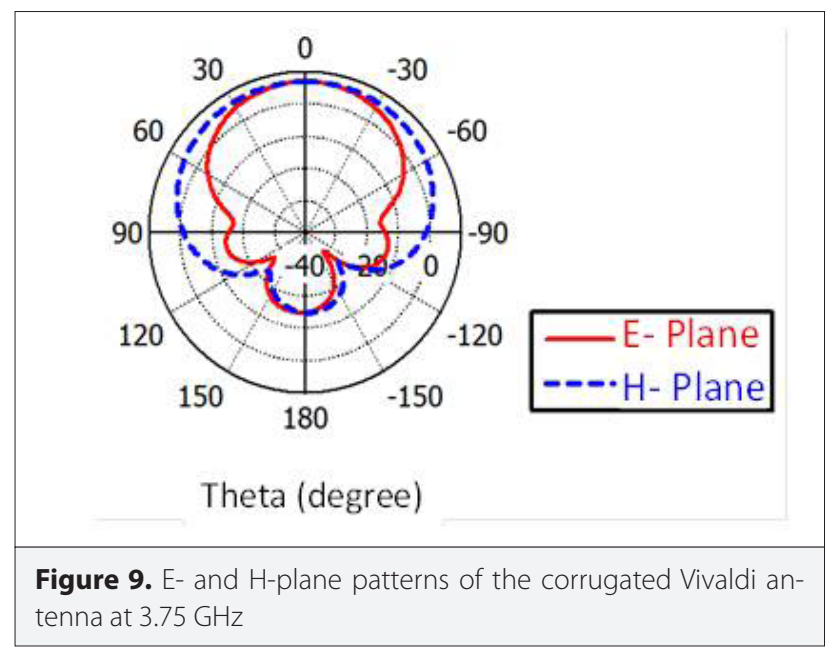

Figure 9. E- and H-plane patterns of the corrugated Vivaldi antenna at $3.75 \mathrm{GHz}$

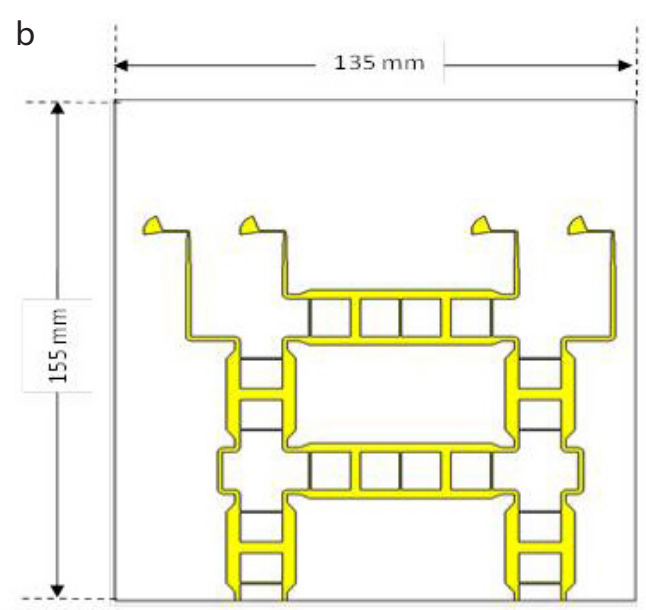

Figure 10. a, b. Four-element corrugated Vivaldi array fed by the Butler matrix: (a) top view and (b) bottom view 


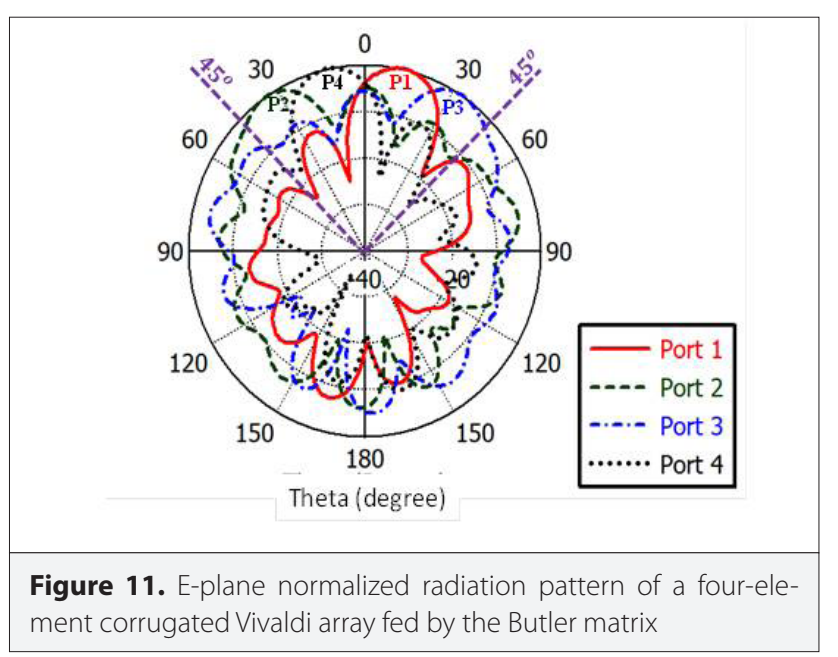

dium frequencies and is simulated with a full-wave simulation tool. Simulation results showed the amplitudes between $-6 \mathrm{~dB}$ and $-8 \mathrm{~dB}$ at the outputs of the Butler circuit. Output phases of the Butler circuit can be controlled by the use of phase shifters for beam steering. The Butler circuit is fabricated and measured. The measurement results show similarity with the simulation results. In addition, with an application example on the usage of Butler circuit as the feed network of a four-element linear array, the beam-steering capability of the designed circuit is demonstrated.

Peer-review: Externally peer-reviewed.

Conflict of Interest: The author have no conflicts of interest to declare.

Financial Disclosure: This work was supported by Research Fund of the Yıldız Technical University (Project Number: FBA-2017-3071).

\section{References}

1. White paper, Spirent Communications, "TD-LTE and MIMO Beamforming: Principles and Test Challenges", August 2012.

2. C. Kang, "MIMO beamforming and its impact on testing TD-LTE", Microwave Journal, vol. 55, no. 2, 2012.

3. The International Telecommunication Union Radiocommunication (ITU-R), "IMT vision - Framework and Overall Objectives of the Future Development of IMT for 2020 and Beyond", Rec. ITU-R M.2083-0, pp. 1-21, Sept. 2015.

4. Bilgi Teknolojileri ve İletişim Kurumu (BTK), “5G ve Dikey Sektörler Raporu", Jan. 2018.

5. J. Blass, "Multidirectional antenna-A new approach to stacked beams", IRE International Convention Record, New York, Amerika, 21-25 March 1966, pp. 48-50.

6. S. Mosca, F. Bilotti, A. Toscano, L. Vegni, "A novel design method for Blass matrix beam-forming networks", IEEE Trans. Antennas Propagation, vol. 50, no.2, pp. 225-232, Feb. 2002. [CrossRef]

7. J. Nolen, "Synthesis of Multiple Beam Networks for Arbitrary Illuminations", PhD Thesis, The Johns Hopkins University, Baltimore, USA, 1965.
8. T. Djerafi, N. J. G. Fonseca, K. Wu, "Broadband substrate integrated waveguide $4 \times 4$ Nolen matrix based on coupler delay compensation", IEEE Trans Microw Theory Techn, vol. 59, no.7, pp. 1740-1745, July 2011. [CrossRef]

9. J. Butler, R. Lowe, "Beam forming matrix simplifies design of electronically scanned antennas", Electron Des, vol. 9, pp. 170-173, April 1961.

10. C.W.Wang, T. G. Ma, C. F. Yang, "A new planar artificial transmission line and its applications to a miniaturized butler matrix", IEEE Trans Microw Theory Techn, vol. 55, no. 12, pp. 2792-2801, Dec. 2007. [CrossRef]

11. T. H. Lin, S. K. Hsu, T. L. Wu, "Bandwidth enhancement of $4 \times 4$ Butler matrix using broadband forward-wave directional coupler and phase difference compensation", IEEE Trans Microw Theory Techn, vol. 61, no. 12, pp. 4099-4109, 2013. [CrossRef]

12. S. Karamzadeh, V. Rafii, M. Kartal, B. S. Virdee, "Compact and broadband $4 \times 4$ SIW Butler matrix with phase and magnitude error reduction", IEEE Antennas Wireless Propag Lett, vol. 25, no. 12, pp. 772-774, 2015. [CrossRef]

13. Y. S. Lin, J. H. Lee, "Miniature Butler matrix design using glass based thin-film integrated passive device technology for $2.5-\mathrm{GHz}$ applications", IEEE Trans Microw Theory Techn, vol. 61, no. 7, pp. 2594-2602, 2013. [CrossRef]

14. H. N. Chu, T. G. Ma, "An extended $4 \times 4$ Butler matrix with enhanced beam controllability and widened spatial coverage", IEEE Trans Microw Theory Techn, vol. 66, no. 3, pp. 1301-1311, March 2018. [CrossRef]

15. N. A. Muhammad, S. K. A. Rahim, N. M. Jizat, T. A. Rahman, K. G. Tan, A. W. Reza, "Beam Forming Networks Using Reduced Size Butler Matrix", Wireless Pers Commun., vol. 63, pp. 765-784, Oct. 2012. [CrossRef]

16. T. K. G. Kwang, P. Gardner, " $4 \times 4$ butler matrix beam forming network using novel reduced size branchline coupler", in 31st European Microwave Conference, London, England, 24-26 Sept. 2001. [CrossRef]

17. I. Sakagami, M. Haga, T. Munehiro, "Reduced branch-line coupler using eight two-step stubs", IEE Proc-Microw Antennas Propagat, vol.164, no.6, pp. 455-460, Dec. 1999. [CrossRef]

18. L. Yang, G. B. Giannakis, "Ultra-wideband communications - an idea whose time has come", IEEE Signal Processing Magazine, vol. 21, no.6, pp. 26-54, Nov. 2004. [CrossRef]

19. L. Chiu, Q. Xue, “Wideband parallel-strip $90^{\circ}$ hybrid coupler with swap", Electronics Letters, vol. 44, no. 11, pp. 687-688, May 2008. [CrossRef]

20. J. Yao, C. Lee, S. Yeo, "Microstrip branch-line couplers for crossover application", IEEE Trans Microw Theory Tech, vol. 59, no. 1, pp. 8792, Jan. 2011. [CrossRef]

21. G. K. Pandey, H. S. Singh, P. K. Bharti, A. Pandey, M. K. Meshram, "High gain vivaldi antenna for radar and microwave ımaging applications", Int Journal of Signal Processing Systems, vol. 3, no.1, pp. 35-39, June 2015. [CrossRef]

22. G. Gopikrishnan, Z. Akhterand, M. J. Akhtar, "A novel corrugated four slot Vivaldi antenna loaded with metamaterial cells for microwave imaging", in Asia-Pacific Microwave Conference (APMC), New Delhi, India, 5-9 December 2016. [CrossRef]

23. M. Abbak, M. N. Akıncı, M. Çayören, İ. Akduman, "Experimental microwave imaging with a novel corrugated Vivaldi antenna", IEEE Trans. on Antennas and Propagat., vol. 65, no. 6, pp.3302-3307, June 2017. [CrossRef] 


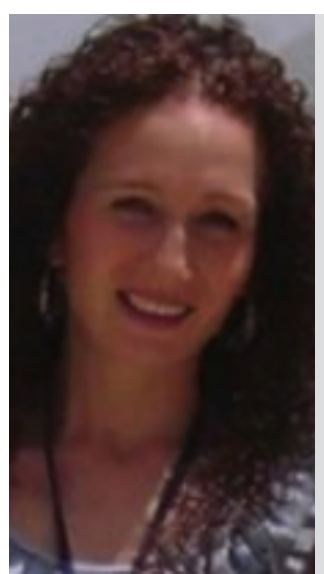

Nurhan Türker Tokan received her B.Sc. degree in Electronics and Communications Engineering from Kocaeli University in 2002 and her M.Sc. and PhD degree in Communication Engineering from Yıldız Technical University (YTU), Istanbul, Turkey, in 2004 and 2009, respectively. From May 2003 to May 2009, she worked as a research assistant in the Electromagnetic Fields and Microwave Technique Section of the Electronics and Communications Engineering Department of YTU, Istanbul, Turkey. Between May 2009 and April 2015, she worked as an assistant professor in the Electronics and Communications Engineering Department of YTU. Since April 2015, she has been working as an associate professor at the same department. From October 2011 to October 2012, she was Postdoctoral researcher in the EEMCS Department of Delft University of Technology, Delft, Netherlands. From October 2012 to May 2013, she was a Postdoctoral Fellow supported by European Science Foundation at the Institute of Electronics and Telecommunications (IETR), University of Rennes 1, Rennes, France. She is the author or coauthor of more than 50 papers published in peer-reviewed international journals and conference proceedings. Her current research interests are analysis and design of antennas with emphasis on dielectric lens antennas and wideband antennas, microwave circuits and intelligent systems. 